\title{
Senescence of T Lymphocytes: Implications for human immunity
}

Arne N. Akbar ${ }^{1}$, Sian M. Henson ${ }^{2}$ and Alessio Lanna ${ }^{1,3}$

${ }^{1}$ Division of Infection and Immunity, University College London.

${ }^{2}$ William Harvey Research Institute, Barts \& The London School of Medicine and Dentistry

Queen Mary, University of London, London.

${ }^{3}$ Nuffield Department of Medicine, University of Oxford, Oxford. United Kingdom.

Correspondence: Professor Arne N. Akbar, Division of Infection and Immunity, The Rayne Building, 5 University Street, London WC1E 6JF.

E-mail.akbar@ucl.ac.uk

\section{Introduction}

As humans continue to live longer, a central concern is to find ways to maintain their health during the extra years of life. Immunity declines during ageing and this is highlighted by the increased susceptibility to infection induced by previously encountered [1,2] as well as new emerging pathogens [3-5] and also decreased ability to respond to vaccination [6]. It is therefore crucial to understand mechanisms that are responsible for this age-associated decrease in immunity and to develop new strategies to enhance immune function to reduce infections and the related consequences of reduced health in older humans. In this article, we discuss how the induction of senescence alters the function of leukocytes, in particular T cells. An emerging concept is that senescence and nutrient sensing signalling pathways within $\mathrm{T}$ cells converge to regulate functional responses. Furthermore the inhibition of these signalling pathways can enhance $T$ cell function is a reason to be optimistic in the search of ways to enhance immunity during ageing. 


\section{What is senescence?}

The word senescence is defined by the Oxford English Dictionary as "The process or condition of growing old ". Cellular senescence was first described in fibroblasts by Leonard Hayflick who with co-workers showed that fibroblasts have a finite capacity to replicate when cultured in vitro, after which they stop dividing but remain metabolically active for extended periods [7]. This constraint on the excessive proliferative activity, i.e. "growing old" of fibroblasts in tissue culture, is known as the Hayflick Limit, that is widely considered to be an anti-cancer mechanism [8]. It was subsequently shown that the molecular basis for the Hayflick Limit is the erosion of telomeres, repeating hexameric DNA sequences at the ends of chromosomes, that occurs each time a cell divides and has been described extensively elsewhere [9-11]. Short telomeres are interpreted as DNA damage by cell sensing machinery that initiates a cascade of events that culminates in growth arrest (reviewed in Akbar and Henson 2011 [12]). Cellular senescence can also result independently of telomere attrition, when there is actual damage to DNA that can occur anywhere in the genome and this also activates a brake on cellular proliferation [13, 14]. The intracellular sensing of DNA damage leads to the initiation of DNA repair, which, if successful, enables the cell to continue its existence. If the DNA cannot be repaired, DNA damage repair foci persist within the nucleus, leading ultimately to growth arrest or senescence. The molecules involved in the DNA repair and the signaling processes that shut down cellular proliferation have been described in detail previously $[15,16]$.While the limit to the extent of proliferation of cells that have damaged DNA is considered to be a natural defense against malignancy, the accumulation of senescent cells is associated with the ageing of tissues [17, $18]$. 
In this article, we will highlight recent information on the signalling processes that are involved in senescence associated proliferative arrest in $\mathrm{T}$ cells, consider the potential impact of accumulation of senescent $\mathrm{T}$ cells on immunity during ageing and discuss ways by which proliferative activity may be reconstituted in these cells with the aim of enhancing immunity.

\section{What is T cell senescence?}

There is a central role for proliferation of antigen-specific $T$ and $B$ cells in the maintenance of immune memory $[19,20]$. The restriction to the extent of proliferation of T cells, especially those that are specific for frequently encountered or persistent antigens may therefore compromise the maintenance of specific immunity in older humans as they may be driven towards senescence [1, 12]. Furthermore, decreased proliferative activity of $\mathrm{T}$ cells after vaccination will reduce vaccine efficacy that is known to be suboptimal in older subjects [6]. However, there is confusion as to what senescence in the immune system actually refers to. In some studies the overall decline in immune function during ageing that involves both the innate and adaptive arms of the immune system, has been termed "immunosenescence" [21]. However, this term does not address specifically the characteristics of individual lymphocytes, and it is a misconception to assume that the function of all leukocytes are decreased during ageing. In this article and in other studies [22-25], T cell senescence refers to the low proliferative activity that results from excessive telomere erosion or DNA damage.

Senescent fibroblasts that are generated by continuous culture until they reach the Hayflick limit stop proliferating but are still metabolically active and perisist as long as they are supplied with nutrients in vitro $[7,26]$. These cells have short telomeres, high activity of the enzyme $\beta$-galactosidase, show high expression of the DNA repair complex associated protein $\gamma \mathrm{H} 2 \mathrm{Ax}$ and constitutive activation of p53 and p38 map kinase (p38 MAPK), as well the cyclin inhibitor 
p16 $[9,27]$ (Table 1). Within primary human $\mathrm{T}$ cells, in both $\mathrm{CD}^{+}[23,28,29]$ and $\mathrm{CD}^{+}[25,30]$ populations, there is a subset of poorly proliferative cells that exhibit characteristics that are very similar to senescent fibroblasts (Fig. 1). These cells can be identified by surface marker expression $\left(\mathrm{CD} 27^{-}, \mathrm{CD} 28^{-}, \mathrm{CD} 4 \mathrm{RA}^{+}, \mathrm{KLRG}^{+}, \mathrm{CD} 57^{+}\right)$that are all characteristics of highly differentiated $T$ cells $[23,28,30,31]$. Unlike senescent fibroblasts however, $T$ cells that have characteristics of senescence are prone to apoptosis in vitro $[23,28]$ although they may be induced to persist by appropriate signals such as growth factors in vivo [32]. An important observation is that $\mathrm{T}$ cells within both $\mathrm{CD}^{+}$and $\mathrm{CD} 8^{+}$compartments that have senescent characteristics increase during ageing [25, 33-38] and are therefore likely to have an impact on altered immune function in older individuals. Furthermore, persistent infection with viruses including cytomegalovirus (CMV) and human immunodeficiency virus (HIV) can induce the accumulation of senescent $\mathrm{T}$ cells with all the characteristics described above, compared to age matched seronegative donors [34, 39, 40]. Although this article focuses on the senescence of human $T$ cells, this process may also govern the proliferation and function of other lymphocytes such as B cells and innate lymphoid cells during ageing but this remains to be investigated.

\section{The secretory nature of senescent cells}

An unexpected property of senescent fibroblasts was their ability to secrete a wide array of proinflammatory cytokines including IL-6 and IL-1 and also other biological mediators such as matrix metalloproteinases [41]. This is known as the senescence associated secretory phenotype or SASP and is considered to be important for remodelling of ageing tissues [41, 42]. In addition, the secretion of chemoattractants including IL-8 and MIP- $1 \alpha$ recruit immune cells to ageing tissue [18] and recent evidence indicates that NK cells can recognize and eliminate senescent cells in vivo [43, 44]. Furthermore, the elimination of senescent cells in a progeroid 
mouse model can reduce age related pathology in many tissues [45]. The SASP may therefore also direct immune cells towards senescent cells in tissues to facilitate their removal. Although the growth promoting, tissue remodelling and senescent cell surveillance inducing activity of the SASP may be beneficial to maintain the integrity of ageing tissue, it is also associated with the development of malignancy [46].

Senescent $\mathrm{T}$ cells within both $\mathrm{CD}^{+}$and $\mathrm{CD}^{+}$subsets express high levels of intracellular granules containing the cytotoxic proteins perforin and granzyme B and can mediate spontaneous antigen-specific cytotoxicity against infected target cells [47-49]. In comparison to less differentiated populations, senescent $\mathrm{T}$ cells also secrete high levels of inflammatory cytokines such IFN $\gamma$ and TNF $\alpha$ after short term activation [28, 30, 50] and also matrix metalloproteinase (Henson SM and Akbar AN, unpublished observations). Therefore, senescent T cells may have an adaptation of the SASP as they are non-proliferative but have a high capacity for secreting immune active mediators. While the SASP in fibroblasts that may be involved with tissue re-modelling, the secretory characteristics of senescent $T$ cells are involved with immune effector functions (Table 1). Therefore, cell type-specific secretory characteristics of senescent cells may be specifically adapted to control different biological processes.

\section{How can we detect senescent $T$ cells?}

The emerging importance of $\mathrm{T}$ cell senescence in the maintenance of immunity during ageing and also the role of the immune system in the elimination of senescent cells in tissues has necessitated the development of new technology to identify senescent cells both within and outside the immune system. In T cells, telomere length can be assessed by measuring telomere 
restriction fragments (TRF) after restriction enzyme digestion of DNA and by qPCR (Q-FISH) [51]. However, these techniques are labour intensive, display variation between batches and require large amounts of DNA and prior subset isolation [51]. Combining flow cytometry with fluorescent in-situ hybridisation (flow-FISH) provides a quick and reproducible technique for telomere length analysis using a flurochrome-labelled telomere probe together with surface and intracellular parameter staining of different leukocyte populations $[34,52,53]$. The flow-FISH technique has been refined to enable the investigation of telomere length, surface phenotype and cytokine production in individual T cells [54] within a mixed cell population. This obviates the need for the isolation of specific subsets of cells and increases the extent of information that can be obtained from small samples of blood [54] or other biological samples.

For $\mathrm{T}$ cells in peripheral blood, there is a correlation between the sequential loss of costimulatory receptors on the cell surface and the progressive loss of telomeres. While undifferentiated $\mathrm{T}$ cells that have relatively long telomeres are $\mathrm{CD} 27^{+} \mathrm{CD} 28^{+}$, senescent $\mathrm{T}$ cells with the shortest telomeres are found within the CD27-CD28- population $[22,28,34,54] . \mathrm{CD}^{+}{ }^{+} \mathrm{T}$ cells at an intermediate stage of differentiation are $\mathrm{CD} 27^{-} \mathrm{CD} 28^{+}$, while $\mathrm{CD}^{+} \mathrm{T}$ cells at a similar stage are $C D 27^{+} \mathrm{CD} 28$, and both these populations have intermediate telomere lengths. The highly differentiated CD27-CD28- population of T cells can be further divided and the cells that re-express CD45RA within this subset have multiple characteristics of senescence including loss of telomerase activity, DNA damage, high levels of reactive oxygen species (ROS) and the lowest proliferative capacity [25, 30]. Intriguingly, the CD45RA re-expressing senesecent T cells do not have critically short telomeres suggesting that senescence in these cell may be induced by other mechanisms including DNA damage by increased ROS production [25, 30]. 
To identify senescent cells in histological section by immunocytochemistry or immunofluorescence, the most common markers used are senescence associated $\beta$-galactosidase (SA $\beta$-gal) expression, the cyclin inhibitor $p 16 /$ INK or the DNA damage response protein $\gamma \mathrm{H} 2 \mathrm{AX}$ however these techniques are rarely combined [55]. A new technique for the robust detection of senescent cells combines the staining for DNA Damage foci within telomere [56]. Ultimately, to investigate the targeting of senescent cells within tissues by the immune system a multiparameter panel of markers for the simultaneous identification of both leukocytes as well as the senescent population will be required.

\section{T cell senescence is maintained by active signalling and is reversible}

Until recently, it was not clear if the low proliferative potential and the loss of ability to upregulate telomerase activity after activation was a passive response due cellular dysfunction, or an actively maintained process involving the activation of inhibitory pathways. It was shown that senescent fibroblasts have constitutively activated p38 MAPkinase and the inhibition of this enzyme revives the proliferative activity of these cells $[57,58]$. This was an early indication that senescence was an actively maintained and not passive process. However fibroblasts, unlike $T$ cells, do not exhibit telomerase activity [59]. These observations led to investigation of whether human senescent T cells, that also exhibit constitutive p38 MAPK activation, could be induced to proliferate upon p38 blockade. Using small molecule inhibitors $[23,30]$ or specific inhibitory shRNA [24] it was found that p38 inhibition reconstituted proliferation and also telomerase activity in $\mathrm{T}$ cells after activation. Therefore the reduced proliferative activity in senescent $\mathrm{T}$ cells is an actively maintained process that is induced by the engagement of specific inhibitory signalling pathways. This raised the question of how p38 was activated in senescent $T$ cells in the first place. 
Two separate pathways for p38 MAPK activation in T cells had been previously described [24, 60]. The canonical MAPK cascade relies on a cascade of upstream kinases, activated by cytokines or co-stimulatory receptor engagement, that phosphorylate and activate downstream kinases culminating ultimately in p38 activation. In this context, inflammatory cytokines such as IFN- $\alpha$, can activate p38 in non-senescent $\mathrm{T}$ cells and this can inhibit proliferation and telomerase activity $[29,34,61]$. This raises the question of whether p38 is activated in senescent $\mathrm{T}$ cells by inflammation in vivo? However senescent $\mathrm{T}$ cells lose expression of costimulatory receptors such as CD27 and CD28 that activate the canonical pathway nor do they express the upstream kinases associated with the canonical pathway suggesting that p38 may be activated an alternative mechanism in these cells $[24,60]$.

An alternative p38 activating pathway that requires TCR ligation that induces p38 activity through auto-phosphorylation, independently of upstream canonical MAPK phosphorylation, has been described [60]. However, it was found that senescent $T$ cells do not express several kinases and scaffold molecules that are involved in this alternative p38 activation pathway and are thus unlikely to engage this second pathway for p38 activation [24, 60]. This suggested the existence of a third unique mechanism for the activation of p38 in senescent T cells. This third p38 activation pathway in $\mathrm{T}$ cells was identified recently and involved the activation of a molecular complex that contains AMP-responsive protein kinase (AMPK), a low nutrient and energy sensor, a scaffold molecule TAB 1 and p38 itself [24] (Fig. 2). This complex induces p38 activation through auto-phosphorylation. The critical point is that AMPK is not only activated by sensing low intracellular glucose but also by endogenous DNA damage (senescence) signalling in T cells but also by [24]. This suggests that there is a convergence of senescence and nutrient sensing pathways in T cells to activate p38 via AMPK/TAB1. Correspondingly, the inhibition of 
either AMPK, TAB1 or p38 itself reconstitutes proliferation as well as telomerase activity in the senescent $\mathrm{T}$ cell population [24]. This provides points for intervention, in addition to targeting p38 itself, to enhance the function of senescent $T$ cells. Undoubtedly other pathways that regulate the senescence characteristics of T cells will be identified in due course.

\section{Metabolism of senescent human T cells}

The regulation of senescent $\mathrm{T}$ cell function by senescence and nutrient sensing raises the question of how senescent $T$ cells obtain their energy for survival and secretory functions how additional energy is obtained for proliferation after blocking the p38 pathway. It was shown that human senescent $\mathrm{CD}^{+} \mathrm{T}$ cells preferentially utilise glycolysis to generate ATP, as opposed to the effector memory subset which are much more metabolically flexible and can either glycolysis or oxidative phosphorylation (OXPHOS) for functional activities (Fig. 3). Senescent $\mathrm{CD}^{+} \mathrm{T}$ cells exhibit mitochondrial dysfunction, increased production of reactive oxygen species (ROS) and impaired mitochondrial biogenesis, which may explain their dependence on glycolysis for energy [25]. The lack of mitochondrial biogenesis may well be a consequence of the failure of senescent $\mathrm{T}$ cells to activate mTOR (mammaliam target of rapamycin), a central integrator of immune signalling and cell metabolism [62], as demonstrated by their inability to phosphorylate the mTOCR1 [25] or the mTORC2 complex [63]. mTOR regulates multiple processes, including autophagy, a degradation pathway that removes damaged or unwanted organelles as well as providing metabolites during periods of starvation [64]. It has been postulated that the decline in autophagy during ageing is due to increased mTORC1 activity. However senescent CD8 ${ }^{+} \mathrm{T}$ cells displayed both low autophagic activity $[25,65]$ and also lack mTORC1 activity [25]. This suggests that the decreased autophagy in senescent $\mathrm{T}$ cells is regulated by a different process. Nevertheless, the defective autophagy results in a failure to 
clear the large giant dysfunctional mitochondria that is linked to increased ROS production by senescent $C D 8^{+} \mathrm{T}$ cells [25].

Since the blockade of p38 increases the proliferation of senescent $T$ cells, it raises the question of how the energy for this enhanced activity is derived. It was found that the p38 signalling inhibited autophagy in senescent T cells, a non-canonical mTORC1 independent process, that occurs instead via a mechanism that involved increased trafficking of the autophagy regulating protein Atg9 from the endosomes to the lysosomes [25]. Blocking p38 enhanced autophagy, increased mitochondrial biogenesis, cleared dysfunctional mitochondria and reduced ROS production in senescent $\mathrm{CD} 8^{+} \mathrm{T}$ cells [25] indicating an overall improvement of mitochondrial fitness. However despite the increased mitochondrial function, senescent $T$ cells still preferentially utilize glycolysis and not OXPHOS for increased proliferation after p38 blockade. Furthermore, the recycling of metabolic precursors resulting from increased autophagy and not greater glucose uptake fuels the enhanced proliferative activity of senescent $T$ cells after p38 inhibition. These results reinforce the observations that senescence and nutrient sensing pathways are inextricably linked and control the function of $\mathrm{T}$ cells.

\section{Inflammation and senescence}

Cellular senescence can cause chronic inflammation through the SASP and this chronic nonmicrobial (sterile) inflammation is thought to be the prime cause of many age-related disorders such as atherosclerosis, autoimmunity, cancers and dementias [42]. Recent investigations have been focused on defining the relationship between inflammageing (association between ageing and increased inflammation) and inflammatory disease [66]. Several studies have reported a 
link between senescence-associated inflammation and a 2-4 fold rise in the levels of acutephase markers, such as C-reactive protein, VCAM-1 and IL-6 [67]. Many inflammatory disease states exhibit a shortening of telomeres in peripheral blood leukocytes. A meta-analysis of 27 studies on 13 different cancers found a significant inverse correlation between immune cell telomere length and cancer incidence [68]. In addition, epidemiological studies showed an increased telomere shortening among patients with cardiovascular disease, atherosclerosis, and myocardial infarction [69]. These studies are correlative with no information as to whether telomere shortening increases the disease risk or whether short telomeres are a secondary consequence of chronic systemic inflammation associated with all these diseases [69]. The fact that inflammatory cytokines such as IFN $\alpha$ can inhibit telomerase in T cells and induce telomere erosion in these cells $[29,34,61]$ lends support to the latter possibility. Molecular studies in rheumatoid arthritis have identified alternative defects affecting telomeric stability. It was found that $\mathrm{T}$ cells from rheumatoid patients accumulate DNA double strand breaks in non-telomeric DNA, indicating more generalised abnormalities in sensing, repairing and tolerating broken DNA [67]. Furthermore healthy individuals carrying the HLA-DR4 haplotype share with rheumatoid patients the age-inappropriate telomere loss, implying that there is a genetic mechanisms in the

premature deterioration of chromosomal ends [70]. Collectively, these data suggest that in addition to a link between nutrition and senescence signalling pathways (Figs 2, 3), inflammation related pathways may be a third interacting axis that is involved in controlling of the function of senescent T cells.

\section{Implications of blocking senescence by targeting p38 to enhance immunity}

The emerging picture is that senescence in T cell is actively regulated and that by blocking cell signalling pathways, the inhibition of proliferation and also telomerase activity in these cells can 
be reversed. The question that arises is whether p38 blockade to alter T cell function would be beneficial or dangerous in vivo. One target for reversing senescence in T cells would be the p38 MAP kinase pathway and based on data that has been obtained in vitro, blocking agents to p38 could be used in vivo to enhance certain T cell functions [23-25, 30]. However since the process of cellular senescence is considered to be a mechanism to safeguard against malignancy by preventing the excessive proliferation of cells with either short telomeres or damaged DNA [8], the possible danger is that the inhibition of senescence may promote cancer. Serendipitously, many pharmaceutical companies have already tested small molecule p38 inhibitors in humans in vivo in phase I,II and II trials to block inflammatory cytokine production in diseases that include rheumatoid arthritis, chronic obstructive pulmonary disease and diabetes [71]. Clinical trials with p38 inhibitors in patients indicate two important points; first that it is safe in the short term (weeks) to treat humans in vivo with p38 inhibitors without a risk of malignancy [71]. However senescent T cell function was not tested before or after treatment with these drugs. These trials have thus far been unsuccessful and were discontinued because of hepatotoxicity after long term treatment (>3 months) and also adaptation of cell signalling pathways leading to reduced drug efficacy [72]. Therefore certain drugs that may target $\mathrm{T}$ cell senescence may have already been developed for clinical use and it may be feasible to treat older humans safely, at least in the short term, to boost immunity.

The existence of at least three different p38 activation pathways in T cells ('Canonical', 'Alternative' and the recently identified AMPK/TAB1 mode) indicates that additional points for intervention to block p38 and thus boost immunity. Since p38 is activated through an AMPK/TAB1 dependent pathway in senescent T cells [24], it may be feasible to inhibit target one or other of these molecules to block p38 signalling. However AMPK is also a central 
regulator of cell function and many different cell types including T cells may be affected [73]. Therefore AMPK inhibition may have too broad an effect to be therapeutically viable for enhancing immunity. Nevertheless a drug that is currently being used to treat type II diabetic patients, metformin [74], can activate AMPK although it is not known if this therapy affects T cell function in these patients. Of note, metformin was shown to enhance $\mathrm{T}$ cell memory generation in mice when administered after primary immunization [75]. However, AMPK activation also strongly inhibits effector T cell responses [24]. Thus, AMPK seems to boost memory $\mathrm{T}$ cell generation but may impede effector $\mathrm{T}$ cell responses needed for primary immunisation, which is essential for boosting an immune response in old people i.e. for influenza vaccination. Regarding T cell senescence, a more selective way to inhibit p38 would be to blocking the scaffold molecule TAB1 required for p38 activation in senescent cells, however, further studies are required to indicate the feasibility of this. Moreover, blocking the p38 pathway can only rescue certain features of $\mathrm{T}$ cell senescence. A global approach for boosting immunity by targeting multiple MAP kinases during ageing currently under investigation (Lanna and Akbar, unpublished findings).

\section{Possible situations where inhibiting senescence could enhance immunity}

One situation where blocking the p38 pathway may be advantageous is during vaccination. The efficacy of many vaccines is reduced in older subjects [6] and the short-term blockade of p38 may open a therapeutic window for enhancement of antigen-specific $\mathrm{T}$ cell proliferation, that would increase the number of antigen specific cells. This increase is considered to be one of the end-points of successful vaccination [76]. Another situation for immune enhancement induced by p38 blockade may be in tumour immunotherapy. T cell proliferation and function can also be enhanced by blocking signalling through the inhibitory receptors such as PD-1 [77]. This has 
been exploited in the therapy of tumours, especially melanoma, where PD1 blockade in patients enhances tumour eradication by $\mathrm{T}$ cells and improves the survival of these patients [78]. The pathway involved in PD1 engagement in senescent human T cells is different for that induced by p38 MAP signalling as there is an additive enhancement of proliferation when both PD1 and p38 pathways are blocked simultaneously [30]. Therefore it may be possible to manipulate senescence signalling pathways in conjunction with other signalling pathways to enhance T cell function. While this may be beneficial for older humans, who have decreased overall immunity, it may also be useful for the treatment of patient with malignancy.

It is well recognized that tumours grow even when there are large numbers of $T$ cells surrounding or infiltrating them, indicating impaired T-cell killing activity in situ. Recent studies showed indeed that tumour infiltrating lymphocytes (TILs) possess attributes of glucosedeprived cells resulting in T cell dysfunction [79]. Strikingly, administration of blocking antibodies to CTLA4/PD1 can restore glucose metabolism in mouse TILs contributing to tumour eradication [80]. This indicates that T cell activity is actively inhibited within the tumour microenvironment, in part through the engagement of inhibitory receptor signalling that leads to metabolic remodelling. Another possible mechanism of cancer-mediated immunosuppression is the induction of $\mathrm{T}$ regulatory cells (Tregs), an inhibitory $\mathrm{T}$ cell population, highly represented at the tumour site and characterized by AMPK-dependent metabolic requirements [80]. An exciting possibility for tumour persistence despite the presence of $\mathrm{T}$ cells is that the tumour-driven deprivation of glucose would trigger the AMPK/TAB1 pathway for p38 activation even in nonsenescent T cells. The 'Intra-Sensory' activated p38 would in turn restrict T cell responses. Since senescence and nutrient sensing signals converge to activate AMPK [24], it may be possible to perform nutrient manipulation as well as anti-senescence therapy to promote antitumour activity by T cells. Future work is needed to investigate the role of $T$ cell senescence in cancer, and to characterize the metabolic landscape of TILs especially in humans. 
Nutrient deprivation pathways may also be targetable for boosting responsiveness to vaccination in malnourished individuals, for instance in $3^{\text {rd }}$ world countries. Immune-activation is an energy-demanding process, and it is thus possible that diminished immune function in such individuals may be direct consequence of activation of AMPK-dependent mechanisms. Correspondingly, increased risks for opportunistic infections affect third world populations. This also opens for possible drug-free interventions to boost immune-responsiveness and implement cost-effective, pan-immunization profiles in these people. Simple and immediate interventions, i.e. addition of glucose to vaccine formulations, may unveil potent yet underestimated immunestimulatory effects disrupting AMPK activation and allowing vaccination of malnourished subjects. In the future, exploring nutrient-based strategies and other food-related changes in dietary intake either alone or in combination with drug-based interventions may be a cost effective way to cope with large-scale immunizations.

\section{Future perspectives}

There is an increasing need to understand how cellular processes, that are usually investigated in isolation from each other, are linked. A case in point is the new awareness of the interrelationship between senescence, nutrition and inflammation in regulating $\mathrm{T}$ cell function $[25,29,61]$. Although a supramolecular complex containing AMPK, TAB1 and p38 is involved in regulating the function of senescent $T$ cells, it is possible that this complex also contains other molecules that may be targeted to alter $\mathrm{T}$ cell function. This raises the question of whether other cellular processes also feed into this interactive network. A key unanswered question is whether the same pathways are linked and control the function of other leukocytes and also 
cells outside the immune system in the same way. The fact that telomere erosion in leukocytes correlates with the severity of a vast array of disease states that are associated with many different cell types/organs suggests that this may be the case. A crucial requirement would be the identification of an appropriate animal model for testing interventions, using clinically tested drugs that are already available to target senescent $\mathrm{T}$ cells boost immunity as ethical constraints preclude most of such studies in humans. Nevertheless the key message at present is that the reduced proliferative function of senescent primary human $\mathrm{T}$ cells that accumulate during ageing is globally controlled by divergent interacting pathways that may be reversible. 


\section{References}

1. Akbar, A.N. et al. (2004) Will telomere erosion lead to a loss of T-cell memory? Nat Rev Immunol 4, 737-743

2. Nikolich-Zugich, J. and Rudd, B.D. (2010) Immune memory and aging: an infinite or finite resource? Curr Opin Immunol 22, 535-540

3. Brien, J.D. et al. (2009) Key role of T cell defects in age-related vulnerability to West Nile virus. J Exp Med 206, 2735-2745

4. Whitehorn, J. and Simmons, C.P. (2011) The pathogenesis of dengue. Vaccine 29, 7221-7228

5. Gupta, M. et al. (2004) Persistent infection with ebola virus under conditions of partial immunity. J Virol 78, 958-967

6. Grubeck-Loebenstein, B. et al. (2009) Immunosenescence and vaccine failure in the elderly. Aging Clin Exp Res 21, 201-209

7. Hayflick, L. and Moorhead, P.S. (1961) The serial cultivation of human diploid cell strains. Exp Cell Res 25, 585-621

8. Campisi, J. (2005) Senescent cells, tumor suppression, and organismal aging: good citizens, bad neighbors. Cell 120, 513-522

9. von Zglinicki, T. et al. (2005) Human cell senescence as a DNA damage response. Mech Ageing Dev 126, 111-117

10. Blasco, M.A. (2002) Telomerase beyond telomeres. Nat Rev Cancer 2, 627-633

11. Weng, N.P. (2008) Telomere and adaptive immunity. Mech Ageing Dev 129, 60-66

12. Akbar, A.N. and Henson, S.M. (2011) Are senescence and exhaustion intertwined or unrelated processes that compromise immunity? Nat Rev Immunol 11, 289-295

13. Plunkett, F.J. et al. (2005) The impact of telomere erosion on memory CD8+ T cells in patients with X-linked lymphoproliferative syndrome. Mech Ageing Dev 126, 855-865

14. Chung, H.K. et al. (2005) Extratelomeric functions of telomerase. Curr Mol Med 5, 233241

15. d'Adda di Fagagna, F. (2008) Living on a break: cellular senescence as a DNA-damage response. Nat Rev Cancer 8, 512-522

16. Ben-Porath, I. and Weinberg, R.A. (2005) The signals and pathways activating cellular senescence. Int J Biochem Cell Biol 37, 961-976

17. Baker, D.J. et al. (2016) Naturally occurring p16(Ink4a)-positive cells shorten healthy lifespan. Nature 530, 184-189

18. van Deursen, J.M. (2014) The role of senescent cells in ageing. Nature 509, 439-446

19. Beverley, P.C.L. (2010) Immune Memory: the Basics and How to Trigger an Efficient Long-Term Immune Memory. J Comp Pathol 142, Supplement 1, S91-S95

20. Ahmed, R. and Gray, D. (1996) Immunological Memory and Protective Immunity: Understanding Their Relation. Science 272, 54-60

21. Franceschi, C. et al. (1999) Biomarkers of immunosenescence within an evolutionary perspective: the challenge of heterogeneity and the role of antigenic load. Exp Gerontol 34, 911-921

22. Plunkett, F.J. et al. (2007) The loss of telomerase activity in highly differentiated CD8+CD28-CD27- T cells is associated with decreased Akt (Ser473) phosphorylation. $J$ Immunol 178, 7710-7719

23. Di Mitri, D. et al. (2011) Reversible Senescence in Human CD4+CD45RA+CD27Memory T Cells. J Immunol 187, 2093-2100

24. Lanna, A. et al. (2014) The kinase p38 activated by the metabolic regulator AMPK and scaffold TAB1 drives the senescence of human T cells. Nat Immunol 15, 965-972 
25. Henson, S.M. et al. (2014) p38 signaling inhibits mTORC1-independent autophagy in senescent human CD8+ T cells. J Clin Invest 124, 4004-4016

26. Blagosklonny, M.V. (2011) Cell cycle arrest is not senescence. Aging 3, 94-101

27. Freund, A. et al. (2010) Inflammatory networks during cellular senescence: causes and consequences. Trends in Molecular Medicine 16, 238-246

28. Libri, V. et al. (2011) Cytomegalovirus infection induces the accumulation of short-lived, multifunctional CD4+CD45RA+CD27+ T cells: the potential involvement of interleukin-7 in this process. Immunology 132, 326-339

29. Lanna, A. et al. (2013) IFN-alpha inhibits telomerase in human CD8(+) T cells by both hTERT downregulation and induction of p38 MAPK signaling. J Immunol 191, 37443752

30. Henson, S.M. et al. (2015) Blockade of PD-1 or p38 MAP kinase signaling enhances senescent human CD8(+) T-cell proliferation by distinct pathways. Eur J Immunol 45, 1441-1451

31. Henson, S.M. et al. (2009) KLRG1 signaling induces defective Akt (ser473) phosphorylation and proliferative dysfunction of highly differentiated CD8+ T cells. Blood 113, 6619-6628

32. Waller, E.C. et al. (2007) Differential costimulation through CD137 (4-1BB) restores proliferation of human virus-specific "effector memory" (CD28(-) CD45RA(HI)) CD8(+) T cells. Blood 110, 4360-4366

33. Appay, V. et al. (2007) Accelerated immune senescence and HIV-1 infection. Exp Gerontol 42, 432-437

34. Fletcher, J.M. et al. (2005) Cytomegalovirus-specific CD4+ T cells in healthy carriers are continuously driven to replicative exhaustion. J Immunol 175, 8218-8225

35. Hamann, D. et al. (1997) Phenotypic and functional separation of memory and effector human CD8+ T cells. J Exp Med 186, 1407-1418

36. Khan, N. et al. (2002) Cytomegalovirus seropositivity drives the CD8 T cell repertoire toward greater clonality in healthy elderly individuals. J Immunol 169, 1984-1992

37. Das, A. et al. (2008) Functional skewing of the global CD8 T cell population in chronic hepatitis B virus infection. J Exp Med 205, 2111-2124

38. Hoare, M. et al. (2010) CD4+ T-lymphocyte telomere length is related to fibrosis stage, clinical outcome and treatment response in chronic hepatitis $\mathrm{C}$ virus infection. $J$ Hepatol $53,252-260$

39. Appay, V. et al. (2008) CD8+ T cell efficacy in vaccination and disease. Nat Med 14, 623-628

40. Hertoghs, K.M. et al. (2010) Molecular profiling of cytomegalovirus-induced human CD8+ T cell differentiation. J Clin Invest 120, 4077-4090

41. Coppe, J.P. et al. (2010) The senescence-associated secretory phenotype: the dark side of tumor suppression. Annual review of pathology 5, 99-118

42. Tchkonia, T. et al. (2013) Cellular senescence and the senescent secretory phenotype: therapeutic opportunities. J Clin Invest 123, 966-972

43. Krizhanovsky, V. et al. (2008) Senescence of activated stellate cells limits liver fibrosis. Cell 134, 657-667

44. Sagiv, A. et al. (2016) NKG2D ligands mediate immunosurveillance of senescent cells. Aging 8, 328-344

45. Baker, D.J. et al. (2004) BubR1 insufficiency causes early onset of aging-associated phenotypes and infertility in mice. Nature genetics 36, 744-749

46. Coppe, J.P. et al. (2008) Senescence-associated secretory phenotypes reveal cellnonautonomous functions of oncogenic RAS and the p53 tumor suppressor. PLoS Biol 6, 2853-2868 
47. Hislop, A.D. et al. (2007) Cellular responses to viral infection in humans: lessons from Epstein-Barr virus. Annu Rev Immunol 25, 587-617

48. Dunne, P.J. et al. (2005) Quiescence and functional reprogramming of Epstein-Barr virus (EBV)-specific CD8+ T cells during persistent infection. Blood 106, 558-565

49. Long, H.M. et al. (2011) Cytotoxic CD4+ T Cell Responses to EBV Contrast with CD8 Responses in Breadth of Lytic Cycle Antigen Choice and in Lytic Cycle Recognition. The Journal of Immunology 187, 92-101

50. Dunne, P.J. et al. (2002) Epstein-Barr virus-specific CD8(+) T cells that re-express CD45RA are apoptosis-resistant memory cells that retain replicative potential. Blood $100,933-940$

51. Montpetit, A.J. et al. (2014) Telomere length: a review of methods for measurement. Nursing research 63, 289-299

52. Baerlocher, G.M. et al. (2006) Flow cytometry and FISH to measure the average length of telomeres (flow FISH). Nature protocols 1, 2365-2376

53. Rufer, N. et al. (2003) Ex vivo characterization of human CD8+ T subsets with distinct replicative history and partial effector functions. Blood 102, 1779-1787

54. Riddell, N.E. et al. (2015) Multifunctional cytomegalovirus (CMV)-specific CD8(+) T cells are not restricted by telomere-related senescence in young or old adults. Immunology $144,549-560$

55. de Jesus, B.B. and Blasco, M.A. (2012) Assessing Cell and Organ Senescence Biomarkers. Circulation research 111, 97-109

56. Hewitt, G. et al. (2012) Telomeres are favoured targets of a persistent DNA damage response in ageing and stress-induced senescence. Nat Commun 3, 708

57. Passos, J.F. et al. (2010) Feedback between p21 and reactive oxygen production is necessary for cell senescence. Mol Syst Biol 6, 347

58. Davis, T. et al. (2010) Evaluating the role of p38 MAP kinase in growth of Werner syndrome fibroblasts. Ann N Y Acad Sci 1197, 45-48

59. Cong, Y.-S. et al. (2002) Human Telomerase and Its Regulation. Microbiology and Molecular Biology Reviews 66, 407-425

60. Salvador, J.M. et al. (2005) Alternative p38 activation pathway mediated by T cell receptor-proximal tyrosine kinases. Nat Immunol 6, 390-395

61. Reed, J.R. et al. (2004) Telomere erosion in memory T cells induced by telomerase inhibition at the site of antigenic challenge in vivo. J Exp Med 199, 1433-1443

62. Powell, J.D. et al. (2012) Regulation of immune responses by mTOR. Annu Rev Immunol 30, 39-68

63. Franzese, O. et al. (2014) Defect in HSP90 expression in highly differentiated human CD8+ T lymphocytes. Cell Death Dis 5, e1294

64. Terman, A. and Brunk, U.T. (2005) The aging myocardium: roles of mitochondrial damage and lysosomal degradation. Heart, lung \& circulation 14, 107-114

65. Phadwal, K. et al. (2012) A novel method for autophagy detection in primary cells: impaired levels of macroautophagy in immunosenescent T cells. Autophagy 8, 677-689

66. Franceschi, C. et al. (2000) Inflamm-aging. An evolutionary perspective on immunosenescence. Ann N Y Acad Sci 908, 244-254

67. Hohensinner, P.J. et al. (2011) Telomere Dysfunction, Autoimmunity and Aging. Aging and disease 2, 524-537

68. Wentzensen, I.M. et al. (2011) The Association of Telomere Length and Cancer: A MetaAnalysis. Cancer Epidemiol Biomarkers Prev 20, 1238-1250

69. Chou, J.P. and Effros, R.B. (2013) T cell replicative senescence in human aging. Current pharmaceutical design 19, 1680-1698 
70. Schönland, S.O. et al. (2003) Premature telomeric loss in rheumatoid arthritis is genetically determined and involves both myeloid and lymphoid cell lineages. Proc Natl Acad Sci U S A 100, 13471-13476

71. Patterson, H. et al. (2014) Protein kinase inhibitors in the treatment of inflammatory and autoimmune diseases. Clinical \& Experimental Immunology 176, 1-10

72. Sweeney, S.E. (2009) The as-yet unfulfilled promise of p38 MAPK inhibitors. Nature reviews. Rheumatology 5, 475-477

73. Ho, P.-C. and Liu, P.-S. (2016) Metabolic communication in tumors: a new layer of immunoregulation for immune evasion. Journal for Immunotherapy of Cancer 4, 4

74. Viollet, B. et al. (2012) Cellular and molecular mechanisms of metformin: an overview. Clinical Science (London, England : 1979) 122, 253-270

75. Pearce, E.L. et al. (2009) Enhancing CD8 T-cell memory by modulating fatty acid metabolism. Nature 460, 103-107

76. Wherry, E.J. et al. (2005) Low CD8 T-cell proliferative potential and high viral load limit the effectiveness of therapeutic vaccination. J Virol 79, 8960-8968

77. Riella, L.V. et al. (2012) Role of the PD-1 Pathway in the Immune Response. Am J Transplant 12, 2575-2587

78. Mahoney, K.M. et al. (2015) Combination cancer immunotherapy and new immunomodulatory targets. Nat Rev Drug Discov 14, 561-584

79. Pearce, E.L. and Pearce, E.J. (2013) Metabolic pathways in immune cell activation and quiescence. Immunity 38, 633-643

80. Ho, P.-C. et al. (2015) Phosphoenolpyruvate Is a Metabolic Checkpoint of Anti-tumor T Cell Responses. Cell 162, 1217-1228 
Figure 1. Differences in the manifestation of senescence in T cells and fibroblasts.

The majority of data on cellular senescence has been performed on fibroblasts, $\mathrm{T}$ cells also undergo senescence in a similar but not identical manner. Both cell types respond similarly to senescence stimuli by inducing a DNA damage response, associated with temporary growth arrest. If this DNA damage cannot be repaired a state of deep irreversible senescence ensues. $\mathrm{T}$ cells however are susceptible to apoptosis and die before they reach this stage whereas fibroblasts can remain for long periods in a non-proliferative state. Reviewed in $[9,15]$.

\section{Figure 2. AMPK activates p38 MAPK in senescent T cells.}

The canonical MAPK cascade is the main mechanism for activation of p38 in mammalian cells, including T cells. However senescent T cells have an engage a distinct mechanism, whereby under low nutrient conditions p38 is phosphorylated via AMPK and the scaffold protein TAB1 $\beta$, an isoform only expressed in senescent T cells.

Figure 3. Senescent T cells lose their metabolic flexibility

T cells can utilise either OXPHOS or glycolysis to meet their energy requirements. However senescent $\mathrm{CD}^{+} \mathrm{T}$ cells display a profound mitochondrial dysfunction causing the cells to switch to glycolysis in order to fuel their effector functions. This metabolic switch renders the senescent $\mathrm{CD}^{+} \mathrm{T}$ cells metabolic unstable. 
Figure 1.

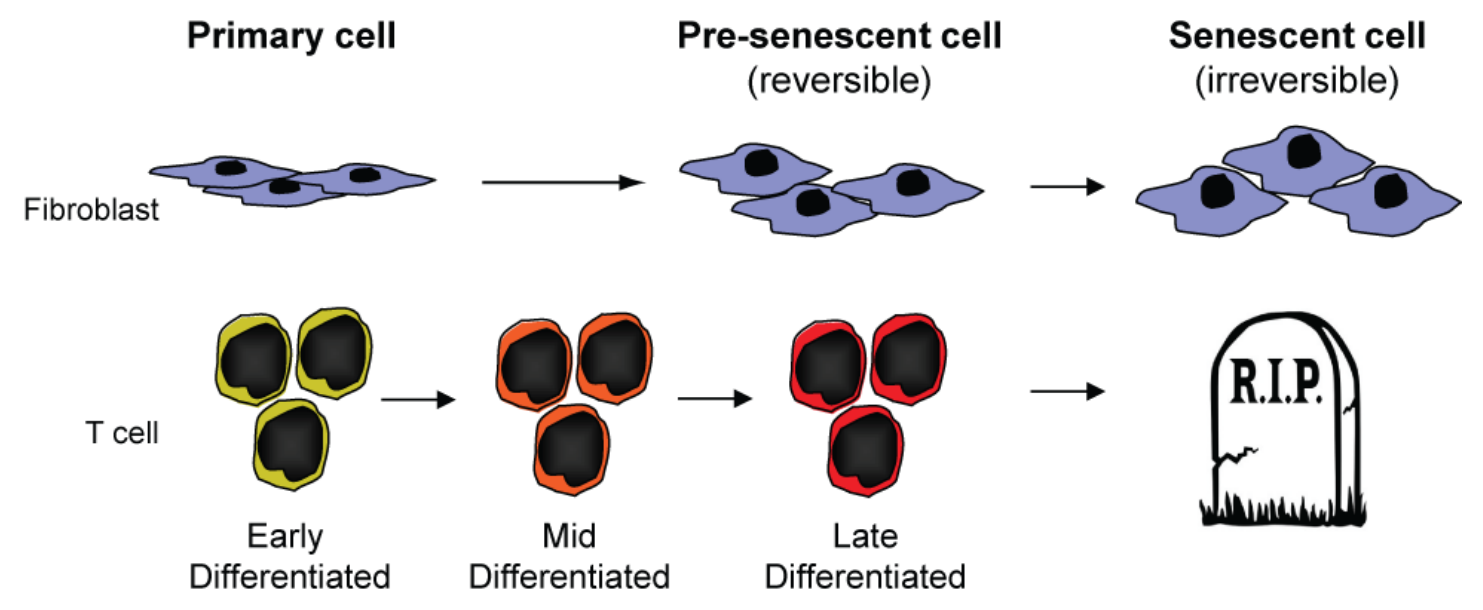


Figure 2.
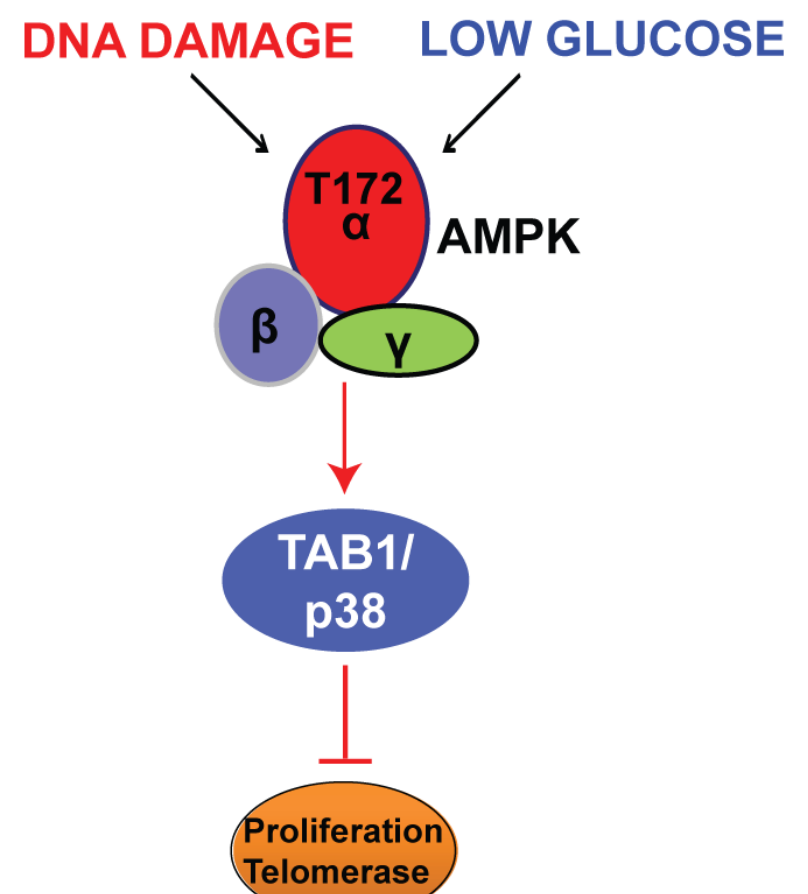
Figure 3.

Non-senescent CD8+ T Cell

Senescent CD8+ T Cell

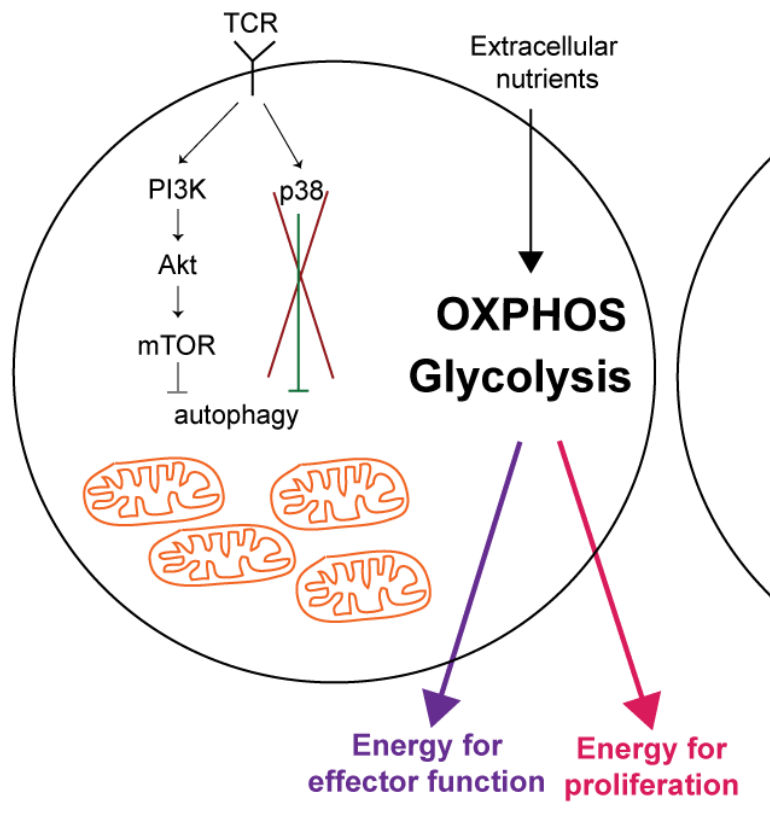

TCR Extracellular

PI31 p38

A kt

$\perp \quad$ Glycolysis

autophagy

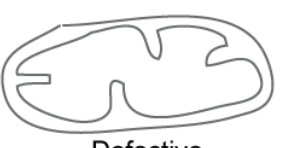

Defective

mitochondria

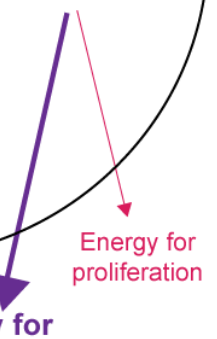

Energy for

effector function 


\section{Table 1. Characteristics of fibroblast vs T cell senescence.}

The majority of data on cellular senescence has been performed on fibroblasts, $\mathrm{T}$ cells also undergo senescence in a similar but not identical manner. The key features of the senescence phenotype are common to fibroblast and $\mathrm{T}$ cells, such as the lack of proliferation, short telomeres and the inability to upregulate telomerase. However a major difference is the susceptibility to apoptosis with fibroblasts being long lived and $\mathrm{T}$ cells succumbing to death by apoptosis. Furthermore it is not known whether senescent T cells express high levels of $\beta$ galactosidase or $\gamma \mathrm{H} 2 \mathrm{AX}$. For reviews see $[1,15]$ 
Table 1.

\begin{tabular}{|lc|}
\hline \multicolumn{2}{|l|}{ Fibroblast Senescence } \\
\hline Proliferation & $\downarrow$ \\
Telomeres & $\downarrow$ \\
Telomerase & $\downarrow$ \\
Apoptosis & $\downarrow$ \\
$\beta$-galactosidase & $\uparrow$ \\
$\gamma \mathrm{H}$ 2AX & $\uparrow$ \\
p16 & $\uparrow$ \\
p53 & $\uparrow$ \\
p38 & $\uparrow$ \\
Cytokine & $\uparrow$ \\
production & \\
\hline
\end{tabular}

\begin{tabular}{|lc|}
\hline \multicolumn{2}{|c|}{ T cell Senescence } \\
\hline Proliferation & $\downarrow$ \\
Telomeres & $\downarrow$ \\
Telomerase & $\downarrow$ \\
Apoptosis & $\downarrow$ \\
$\beta$-galactosidase & $?$ \\
$\gamma \mathrm{H} 2 \mathrm{AX}$ & $\uparrow$ \\
p16 & $?$ \\
p53 & $\uparrow$ \\
p38 & $\uparrow$ \\
Cytokine & $\uparrow$ \\
production & \\
\hline
\end{tabular}

